\title{
An Image Fusion Algorithm Based on Local Laplasse Filter
}

\author{
Jiang shuai ${ }^{1, a}$, Li xiongfei ${ }^{1, b}$, Zhang xiaoli ${ }^{1, c}$ \\ ${ }^{1}$ School of JiLin University, ChangChun 130012, China. \\ a546538097@qq.com, b3712258@qq.com, ${ }^{\text {5 } 543962260 @ q q . c o m ~}$
}

Keywords: Image fusion, multi scale, local Laplasse filter, detail layer, smooth layer, edge information

\begin{abstract}
To deal with the problem of smooth image progressing on the edge, presents a multi scale image fusion algorithm (SLP). The algorithm is based on the local Laplasse filter. First of all, using the local Laplasse filter, the images are decomposed into detail layers and smooth layers. Secondly, the detail layers of the two images are transformed into a fusion rule map by using the sigmoid function, fuse the detail layer according to the map can get the information of the images; Fusing the smooth layers by mean value method can get the picture of the images. Finally, merging the detail layer and smooth layer to generate a clear picture of all the scenes. The algorithm can not only preserve the edge information, but also can reduce the image noise and preserve the image edge details effectively. Experimental results show that the proposed algorithm is superior to Principal Component Analysis(PCA), Ratio pyramid(RP), Morphological pyramid(MP) in terms of performance.
\end{abstract}

\section{Introduction}

Recent years, image fusion technology has played a huge advantage in many fields. Such as: fusing the CT image and nuclear magnetic resonance MRI image [1] to accurate diagnosis of disease accurately; using the infrared images and visible image [2] to help pilots accurate navigation, etc.. With the rapid progress of the information , various image fusion technology will be fully used in different areas, with the development of research and application, the image fusion technology will be more perfect.

According to the different stages of image fusion, it can be divided into 3 levels: pixel level (Pixel-level) image fusion, feature level (Feature-level) image fusion and decision level (Decision-level) image fusion. The pixel level fusion algorithm is used in this paper.

In Pixel level image fusion, the algorithm analyses the signals of sensors directly after preprocess, the accuracy of image registration affects the fusion effect directly. So, before fusing, the image must be registered. There are mainly two kinds of this fusion algorithm : One based on spatial domain and one based on transform domain. The fusion algorithm based on space is a relatively early approach, it is a simple fusion process that operates on the gray value of the image directly without of the process of transformation and inverse transformation. This fusion algorithm includes principal component analysis(PCA) [3],contrast modulation method [4], Dempster-Shafer(D-S) [5], Markov random field(MRF) [6], Bayesian optimization algorithm (BOA) [7,8], double modal neural network method [9]; The fusion algorithm based on transformation has been developed in recent years, it increases the complexity of computation, but it improves the efficiency of image fusion greatly. The main algorithms are: Laplacian Pyramid(LP) [10], Wavelet transform(WT), Contourlet transform method, etc.. This two kinds of fusion algorithm don`t exist independently, and many algorithms combine the two together to achieve the best fusion result.

\section{Laplacian Pyramid algorithm}

In 1983, Burt and Adelson proposed the Laplasse Pyramid algorithm (LPT) [11], and applied it in image decomposition, encoding and reconstruction, etc.. The algorithm using the low pass filter layer to hierarchical images, every layers correspond to a specific scale. The larger the scale is, the 
fuzzier the fine structure in the image will be, but the contour characteristic of the image will also remain, so we can discern features in certain scale.

The Laplasse algorithm can not only be applied in image analysis, but also can be used in the field of compression, texture synthesis and so on. It s more simple because its simple program. However, the general Laplasse algorithm has a disadvantage: in the edge processing which requires high quality such as: edge smoothing and color mapping, The Laplasse algorithm is not a good methord.

\section{Image fusion algorithm based on local Laplasse filter}

Local Laplasse [12] algorithm is mainly used to the image processing for preserving the edge of the smooth, it can effectively reduce the image noise and maintain the image edge details. It combines the position information of the image pixels in the space and the similarity of the pixel value information, so that it has the characteristics of non iterative, local and simple. The two-dimensional weighted coefficient of the local Laplace domain is decided by two parts, one is determined by the spatial distance between pixels; another part is determined by the brightness of the pixel

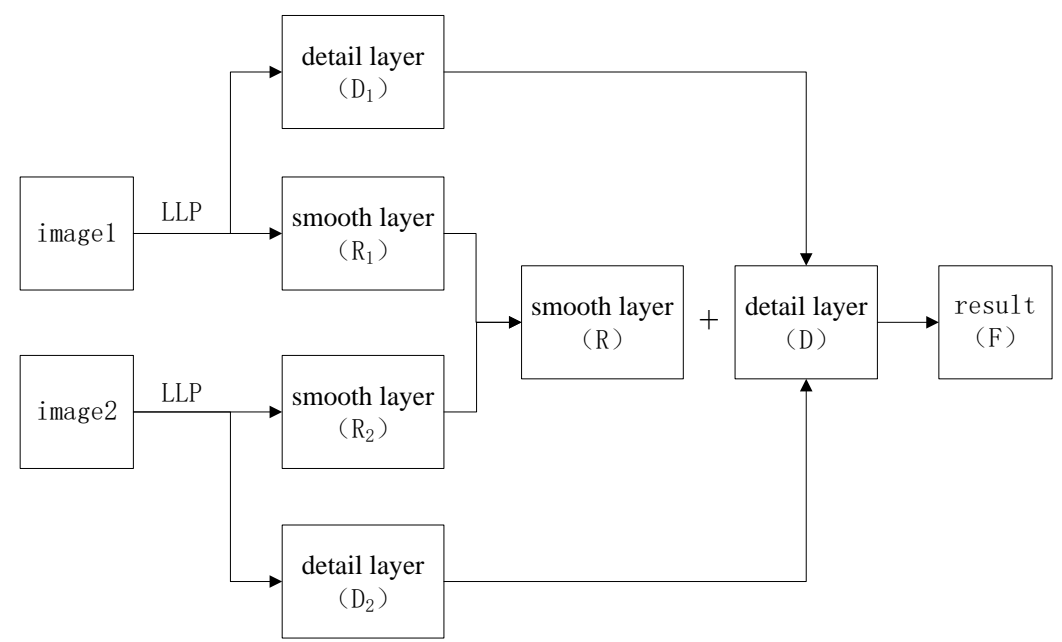

Fig. 1 Flow chart of fusion algorithm

This paper proposes an image fusion algorithm based on local Laplasse filter which is shown in Fig. 1. Assuming the images are $\mathrm{I}_{1}$ and $\mathrm{I}_{2}$, the size of both are $\mathrm{N} * \mathrm{M}$, and they are processed by noise reduction and preprocess. The specific details of the algorithm are as follows:

1) LLP transformation decomposition : Using local Laplacian to decompose $I_{1}$ and $I_{2}$ respectively, we can get the smoothing layers R1,1, R2,1 and the detail layers d1,1, D2,1 of each image matrixes. Doing the local Laplacian decomposition of every smooth layers until it reaches the $\mathrm{K}$ layers

$$
\begin{aligned}
& I_{1} \stackrel{\text { K level LLP }}{\longrightarrow}\left\{D_{1,1}, D_{1,2}, \ldots, D_{1, K}, R_{1, K}\right\} \\
& I_{2} \stackrel{\text { K level LLP }}{\longrightarrow}\left\{D_{2,1}, D_{2,2}, \ldots, D_{2, K}, R_{2, K}\right\}
\end{aligned}
$$

2) Fusion rules: due to the detail and smooth layers have different meanings, we must use different fusion rules to fusion the images in order to separate the information of detail layers and smooth layers.

i) The rule of smoothing layer fusion: the smooth layer is a generalization of the image. Due to the decimation in the process of decomposition, the sub images of the smooth layer are reduced, in order to save more information of the source image, the smoothing layer matrixes are fused by means of the mean value method.

Assuming two smoothing layer matrixes are $R_{1, K}$ and $R_{2, K}$, the smoothed layer matrix 
is $\mathrm{R}$, then we can be obtained:

$$
R=\left(R_{1, K}+R_{2, K}\right) / 2
$$

ii) The rule of detail layer fusion: detail layer matrix retains various features of the image, the large absolute values represent mutations, they may be the edge or texture of the image. This algorithm uses sigmoid excitation function on the detail layer matrix to set the weight: larger matrix numerical get larger weight, on the contrary, the smaller the matrix numerical is the smaller the weight will be.

- Assuming two detail layer matrixes are $\mathrm{D}_{1, \mathrm{i}}$ and $\mathrm{D}_{2, \mathrm{i}}$, and the weight factor of $\mathrm{D}_{1, \mathrm{I}}$ is $b_{i}$, its formula is as follows:

$$
b_{\mathrm{i}}=\frac{1}{1+e^{-\mathrm{a} *\left(\mathrm{D}_{1, \mathrm{i}}-\mathrm{D}_{2, \mathrm{i}}\right)}}
$$

' $a$ ' is a constant coefficient, here it`s 1 . The weight factor is set to $\mathrm{S}$ function to make the fusion result more smooth and natural and also prevent the emergence of artifacts.

- Assuming $D_{F, i}$ is the fused detail layer, we can get:

$$
D_{F, i}=\mathrm{D}_{1, i} * b_{i}+\mathrm{D}_{2, i} *\left(1-b_{i}\right)
$$

The algorithm uses multi-scale decomposition, every decomposition of the detail layer need to be added to the final output, Assuming $\mathrm{D}$ is the final output, we can get:

$$
D=\sum_{i=1}^{K} D_{F, i}
$$

3) The reconstruction of Local Laplasse: according to the smooth layers above we can get the final fusion image $\mathrm{F}$ after reconstruction:

$$
F=D+R
$$

\section{Experiment}

In order to verify the effectiveness of the algorithm, we chose three similar algorithms to compare: principal component analysis (PCA), ratio pyramid (RP) and the Morphological pyramid (MP). In order to evaluate the advantages and disadvantages, we use both subjective and objective evaluation method. In subjective evaluation, we evaluate the algorithm from three aspects : (a). The fused image whether remain the visual information as much as possible; (b). Fusion process whether dopes with other inconsistent information; (c). Fusion algorithm is shift invariance and rotation invariance. In objective evaluation, we use five kinds of objective evaluation index to evaluate the quality of the fused image: $Q^{F}{ }_{A B}[13]$, Piella $\left(Q_{0}, Q_{W}, Q_{E}\right)$ [14] and VIFF[15]。

The experimental results of image fusion in the first groups are shown in Fig. 2, figure 2(a) and figure 2(b) are the two source images to be fused. In figure 2(a) the magnum nearby is relatively clear, the gear and the small bottle faraway are vague, figure 2(b) is opposite. Figure 2(c) - figure 2(f) are the fusion image obtained by different algorithms. The result shows that RP and MP algorithm is not ideal enough: the handwriting is not clear, and the afterimage is obvious; The quality of SLP, PCA are better, but in the vision of the small bottle, PCA still contains afterimage, the edge of the image is not clear.

Table 1 lists the objective evaluation of the first experimental group, the index of the SLP algorithm are marked in bold. The index of $Q^{F}{ }_{A B}, Q_{E}$ in SLP are relatively not very good, but for the other three indicators, SLP algorithm is the best and other algorithms are poor. In general, the SLP algorithm is better than PCA, RP, MP. 


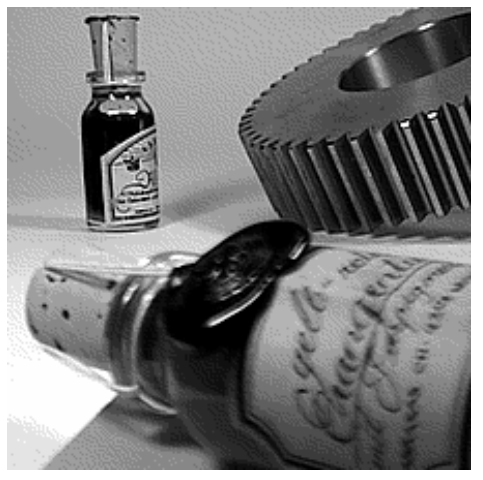

(a) Source Image $I_{1}$

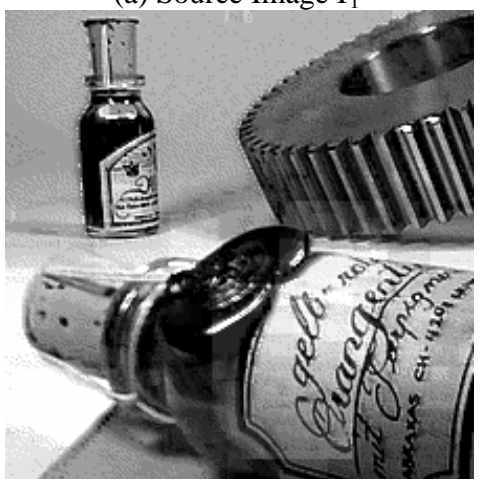

(c) MP

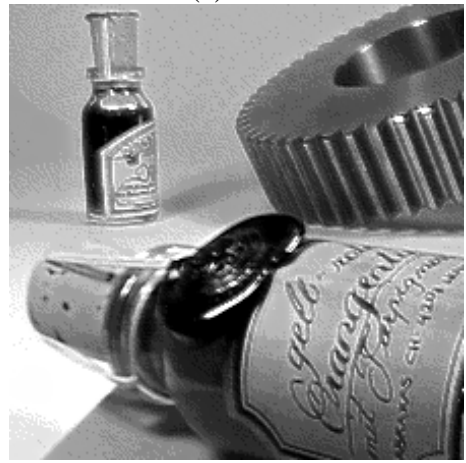

(e) RP

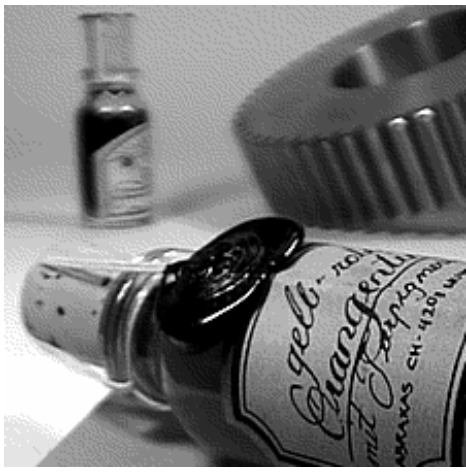

(b)Source Image $I_{2}$

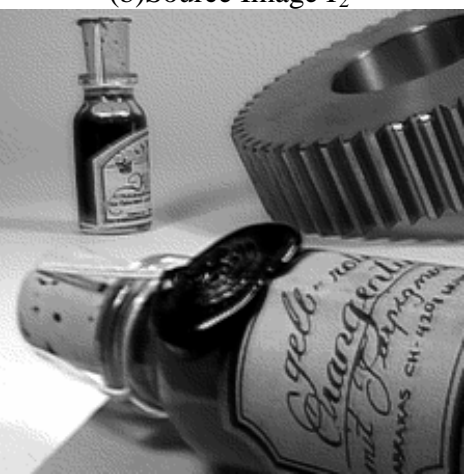

(d) PCA

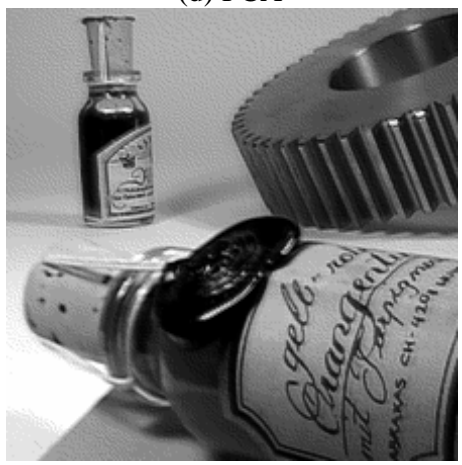

(f) SLP

Fig. 2 The experimental results of the first group

Table 1 The evaluation of the first group

\begin{tabular}{lccccc}
\hline & $Q^{F}{ }_{A B}$ & $Q_{0}$ & $Q_{W}$ & $Q_{E}$ & $V I F F$ \\
\hline PCA & 0.5171 & 0.7143 & 0.7202 & 0.6656 & 0.6906 \\
RP & 0.5806 & 0.8287 & 0.8236 & 0.7353 & 0.7761 \\
MP & 0.5802 & 0.8284 & 0.8234 & 0.7354 & 0.7755 \\
SLP & $\mathbf{0 . 6 0 4 5}$ & $\mathbf{0 . 8 3 4 6}$ & $\mathbf{0 . 8 6 6 1}$ & $\mathbf{0 . 7 2 7 1}$ & $\mathbf{0 . 8 8 7 3}$ \\
\hline
\end{tabular}

Fig. 3 is the experimental results of the second group. In figure 3(a), the small balloon in the right side is relatively clear, the large balloon in the left side is fuzzy, figure 3(b) is on the contrary. The experimental results of figure 3(a) and figure 3(b) are shown in figure 3(c) - figure 3(f).The MP algorithm is obvious fuzzy and the image edge is dark; RP, SLP and PCA performance good, but for the dark hot air balloon in the upper left corner, the markings of the SLP algorithm is better than others.

Table 2 lists the objective evaluation results of the second group. For $Q_{0}, Q_{W}, Q_{E}$ and VIFF, SLP algorithm is the best, Ratio and Morphological are better and PCA is the worstest. 


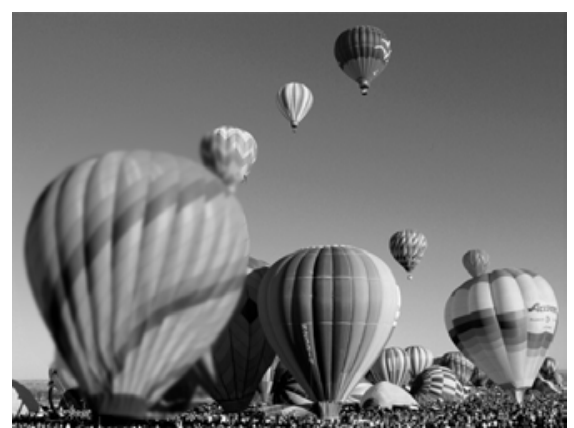

(a) Source Image $\mathrm{I}_{1}$

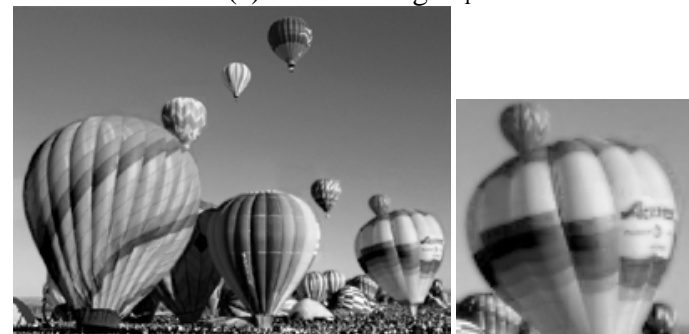

(c) MP

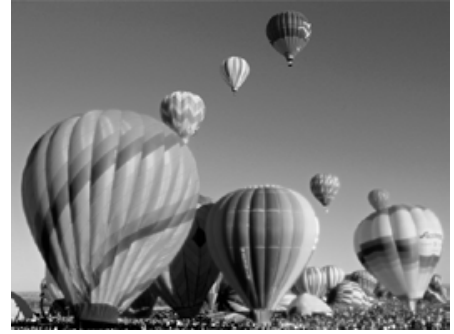

(f) RP

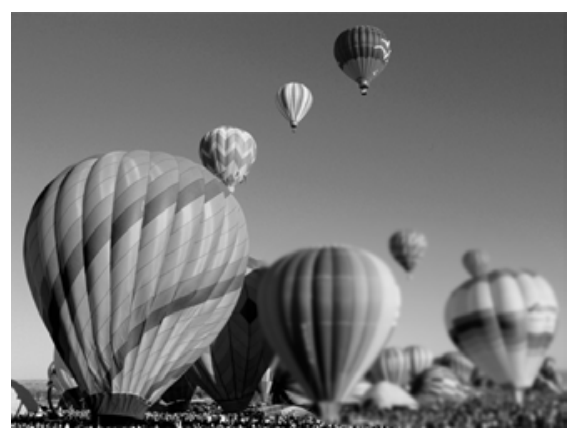

(b)Source Image $I_{2}$

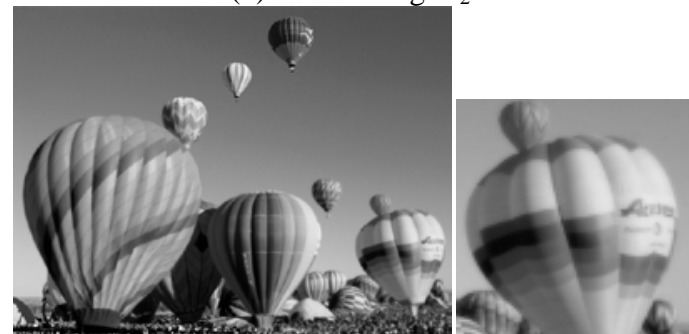

(d) PCA

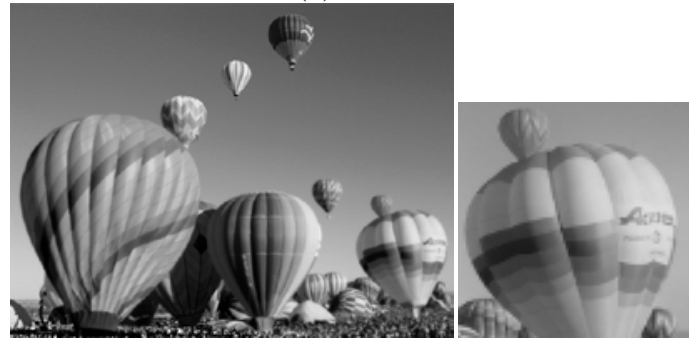

(g) SLP

Fig. 3 The experimental results of the second group

Table 2 The evaluation of the second group

\begin{tabular}{lccccc}
\hline & $Q^{F}{ }_{A B}$ & $Q_{0}$ & $Q_{W}$ & $Q_{E}$ & VIFF \\
\hline PCA & 0.7371 & 0.9361 & 0.8925 & 0.8600 & 0.8461 \\
RP & 0.7643 & 0.9586 & 0.9227 & 0.8847 & 0.8650 \\
MP & 0.7634 & 0.9592 & 0.9224 & 0.8846 & 0.8653 \\
SLP & $\mathbf{0 . 7 4 8 3}$ & $\mathbf{0 . 9 6 8 2}$ & $\mathbf{0 . 9 3 5 3}$ & $\mathbf{0 . 8 9 8 0}$ & $\mathbf{1 . 0 2 5 2}$
\end{tabular}

The experimental results of the third group are shown in Fig. 4. In figure 4(a), the leaves close are clear, the leaves far are fuzzy. The experimental results are shown in figure 4(c) - figure 4(f). The results show that SLP performed better than the other three algorithms: for PCA and RP algorithm, the leaves are unclear which are white; for MP, the image exists dark impurities, and the stems are fuzzy.

Table 3 lists the objective evaluation results of the third group. For $Q_{0}, Q_{W}, Q_{E}$ and VIFF, SLP algorithm is better than other algorithms, then RP, MP and PCA.

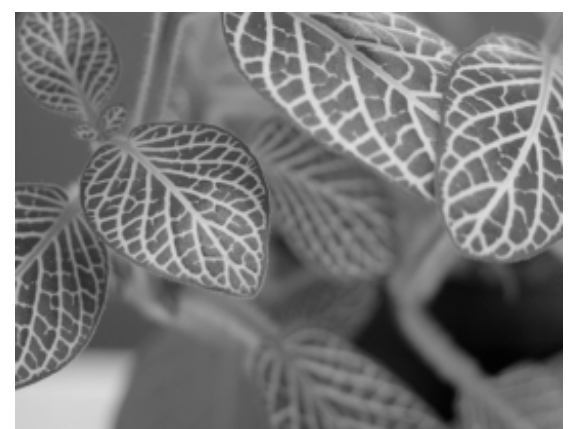

(a) Source Image $I_{1}$

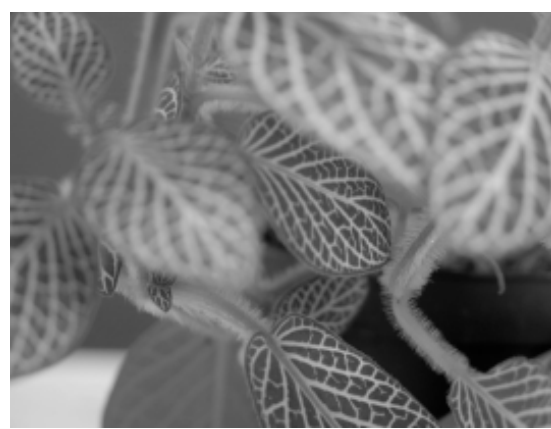

(b) Source Image $\mathrm{I}_{2}$ 


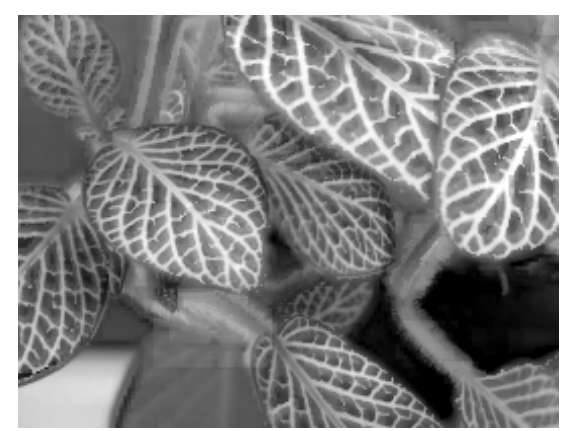

(c) MP

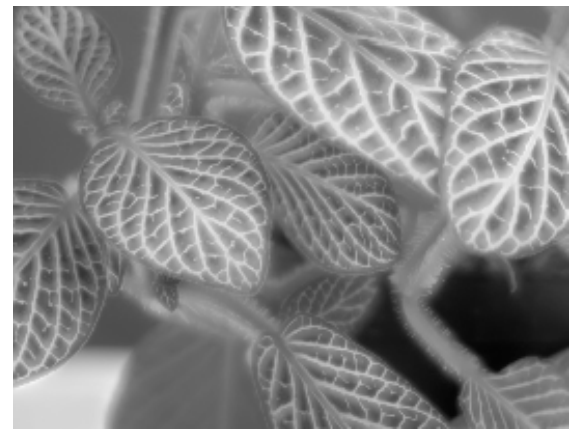

(e) RP

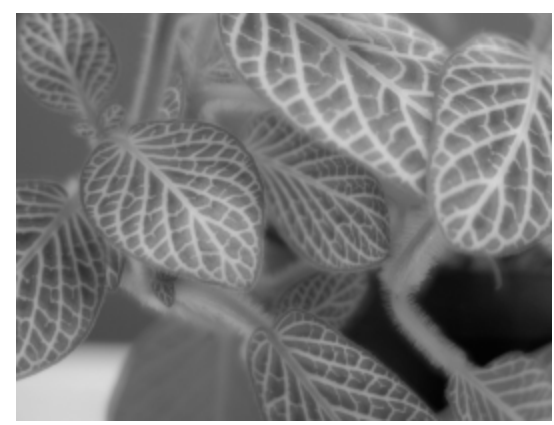

(d) PCA

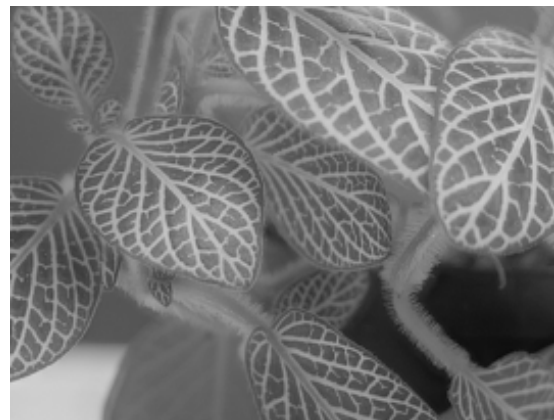

(f) SLP

Fig. 4 The experimental results of the third group

Table 3 The evaluation of the third group

\begin{tabular}{lccccc}
\hline & $Q^{F}{ }_{A B}$ & $Q_{0}$ & $Q_{W}$ & $Q_{E}$ & VIFF \\
\hline PCA & 0.5564 & 0.8051 & 0.7935 & 0.6016 & 0.7877 \\
RP & 0.5457 & 0.8301 & 0.8122 & 0.6343 & 0.7166 \\
MP & 0.5430 & 0.8314 & 0.8118 & 0.6328 & 0.7159 \\
SLP & $\mathbf{0 . 6 7 6 5}$ & $\mathbf{0 . 7 4 5 3}$ & $\mathbf{0 . 8 2 0 9}$ & $\mathbf{0 . 6 4 4 6}$ & $\mathbf{1 . 0 9 9 0}$ \\
\hline
\end{tabular}

\section{Summary}

As a multi-scale image fusion algorithm, Local Laplasse can effectively remain the image features and the effective information, it can also avoid fuzzy images. Therefore, applying the local Laplasse to image fusion can make the image more abundant and comprehensive. At the same time, transform the detail layers and smooth layers of the image according to different frequency is the key to obtain a high quality image. In this paper, we combine the local Laplasse transform and fusion rules, then the experimental results show that this new algorithm is effective and it`s fusion effect is better.

Although the local Laplace transform is mature enough, but how to determine the fusion rules according to the characteristics of images, and how to realize the adaptive fusion, and how to improve the edge sharpness will be the future direction.

\section{References}

[1] Pappas I. P., Puja Malik, Martin Styner, et al. New Method to Assess the Registration of CT-MR Images of the Head[C]. Proceedings of SPIE, 2004, 5370:129-136.

[2] Toet A., Ijspeert J. K., Waxman A. M., et al. Fusion of Visible and Thermal Imagery mproves Situational Awareness [C]. Proceedings of SPIE on Enhanced and Synthetic Vision, 1997,vo1.3088: 177-188.

[3] Smith M I, Heather J P. A review of image fusion technology in 2005[C].Defense and Security. International Society for Optics and Photonics, 2005: 29-45.

[4] Geng B. Y., Xu J. Z., Yang J.Y. An approach based on the features of space-frequency domain 
for fusion of edge maps obtained through multisensors [J]. Systems Engineering and Electronics. 2002, 22(4): 18-22.

[5] J.M.Lafert, F.Heitz, P.Perez et al..Hierarchical statistical models for the fusion of multiresolution image data[C].Proceedings of the International Conference on Computer Vision.Cambridge,USA.1995.908-913.

[6] W A Wright, F Bristol. Quick Markov random field image fusion[C].Proceedings of SPIE, 1998, 3374:302-308.

[7] Sharma R.K.,Leen T.K.,Pavel M. Bayesian sensor image fusion using local linear generative models[J]. Optical Engineering,2001,40(7):1364-1376.

[8] R.K.Sharma,M.Pavel.Adaptive and statistical image fusion[J].Society for Information Display,1996.XXVII:969-972.

[9] Gail A Carpenter, Siegfried Martens, Ogi J Ogas. Self-organizing information fusion and hierarchical knowledge discovery: a new framework using ARTMAP neural networks[J]. Neural Networks,2005,18(3): 287-295.

[10] Toet A.Image fusion by a ratio of low-pass pyramid.Pattern Recognition Letters, 1989, 9(4):245-253.

[11] Perer J.Burt .The Laplacian Pyramid as a Compact Image Code[J], IEEE, 1983,532-540.

[12] Sylvain Paris, Samuel W. Hasinoff, Jan Kautz. Local Laplacian Filters: Edge-aware Image Processing with a Laplacian 2011 , http://people.csail.mit.edu/sparis/publi/2011/siggraph/.

[13] C. S. Xydeas, and V. Petrovic.Objective image fusion performance measure[C], 2000, 36(4):308-309.

[14] G. Piella, and H. Heijmans. A new quality metric for image fusion[C], 2003 International Conference on Image Processing,2003,Vol 3, 173-176.

[15] Y. Han, Y. Cai, Y. Cao et al.A new image fusion performance metric based on visual information fidelity[C],Information fusion, 2013,14(2),127-135. 